Hautarzt 2020 $\cdot 71: 374-379$

https://doi.org/10.1007/s00105-020-04561-y

Online publiziert: 6. März 2020

(c) Der/die Autor(en) 2020

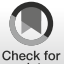

B. Hackenberg $\cdot$ 0. N. Horváth ${ }^{2} \cdot$ M. Petachti ${ }^{3} \cdot$ R. Schult ${ }^{4} \cdot$ N. Yenigün ${ }^{5}$. P. Bannenberg ${ }^{6}$

${ }^{1}$ Hautarztpraxis Dr. Siebenhaar, Solingen, Deutschland

${ }^{2}$ Klinik und Poliklinik für Dermatologie und Allergologie, Ludwig-Maximilians-Universität, München, Deutschland

${ }^{3}$ Klinik für Dermatologie und Allergologie, Vivantes Klinikum Spandau, Berlin, Deutschland

${ }^{4}$ Hautarztpraxis Dr. Brinkmann, Schult \& Samimi-Fard, Gladbeck, Deutschland

${ }^{5}$ Hautarztpraxis N. Yenigün, Kiel, Deutschland

${ }^{6}$ InfectoPharm Arzneimittel und Consilium GmbH, Heppenheim, Deutschland

\title{
Skabiestherapie in Deutschland
}

\section{Ergebnisse einer bundesweiten Umfrage mit besonderem Fokus auf die Wirksamkeit der Erstlinientherapie mit Permethrin}

Seit einigen Jahren wird in Deutschland von vielen dermatologischen Praxen und Klinikambulanzen überregional über eine Häufung von Skabiesfällen berichtet. $\mathrm{Da}$ außerhalb von Ausbrüchen in Gemeinschaftseinrichtungen derzeit keine Meldepflicht nach Infektionsschutzgesetz (IfSG) besteht, fehlen aktuelle und belastbare epidemiologische Zahlen zu Prävalenz und Inzidenz [1], jedoch sprechen neben den Rückmeldungen aus der Praxis auch stetig steigende Verordnungszahlen für eine deutliche Zunahme der Skabies in Deutschland. So sind alleine im Zeitraum 2012 bis 2018 die Verordnungen von Antiskabiosa um das 8-Fache angestiegen [2]. Therapie der ersten Wahl gemäß Leitlinie ist bei gewöhnlicher Skabies in Deutschland die topische Einmalbehandlung mit Permethrin 5\% Creme [1]. Mit steigender Zahl der Skabiesfälle gab es in den letzten Jahren jedoch vermehrt Meldungen, die über ein Nachlassen der Wirksamkeit der Standardtherapie im ärztlichen Alltag berichteten [3]. Eine im November 2016 veröffentlichte systematische Literaturübersicht zur Wirksamkeit und Sicherheit eingesetzter Antiskabiosa bestätigte indes eine anhaltend hohe Heilungsrate für Permethrin

Die Autoren B. Hackenberg, O.N. Horváth, M. Petachti, R. Schult und N. Yenigün trugen in gleichem Ausmaßzu dieser Arbeit bei.
$5 \%$ Creme und zeigte auch im Vergleich zu systemisch angewendetem Ivermectin eine vergleichbar gute Wirksamkeit [4]. Um ein umfassenderes Verständnis von der Wirksamkeit der Erstlinientherapie mit Permethrin in der Praxis zu entwickeln und die Berichte nachlassender Wirksamkeit besser einzuordnen, wurde im September 2018 eine bundesweite Umfrage zur Skabiestherapie mit besonderem Fokus auf die Behandlung mit topischem Permethrin unter dermatologisch tätigen Ärzten in Kliniken und Praxen durchgeführt.

\section{Methodik}

Die Umfrage wurde postalisch versendet und richtete sich an alle in Deutschland dermatologisch tätigen Ärzte [5]. Die Teilnehmer bewerteten mittels eines ungestützten, 8 Fragen umfassenden Fragebogens verschiedene Punkte zur Skabiestherapie mit Permethrin und anderen Antiskabiosa anhand ihrer Erfahrung aus der Praxis. Der Fragebogen beinhaltete Fragen zur wöchentlichen Patientenzahl (1 Frage), zum bevorzugten Diagnoseverfahren (1 Frage), zur individuellen Standardinitialbehandlung (1 Frage), der subjektiv wahrgenommenen Wirksamkeit der Skabiestherapie mit Permethrin (1 Frage), zur Häufigkeit und den (vermuteten) Gründen für ein mögliches Therapieversagen unter Permethrin-Be- handlung (2 Fragen) und zum weiteren therapeutischen Vorgehen bei Versagen der Initialtherapie (2 Fragen). Die von den Befragten angegebene Wirksamkeit der Permethrin-Behandlung wurde zusätzlich anhand der Postleitzahlen (PLZ) geografisch ausgewertet, um mögliche regionale Unterschiede innerhalb Deutschlands aufzeigen zu können. Teilnehmer, die die wahrgenommene Wirksamkeit der Therapie initial nicht in Prozentzahlen angegeben hatten, wurden erneut kontaktiert und um eine prozentuale Angabe gebeten. Diese wurden anschließend wie folgt kategorisiert: sehr gut $(80-100 \%)$, gut $(60-80 \%)$, mäßig (40-60\%), gering (20-40\%), sehr gering (0-20\%).

\section{Ergebnisse}

\section{Teilnehmerkollektiv}

Insgesamt wurden 7651 dermatologisch tätige Ärzte in Deutschland angeschrieben und befragt; 187 Ärzte $(2,4 \%)$ aus insgesamt 75 der bundesweit 95 PLZ-Regionen (78,9\% Abdeckung, keine Rückmeldungen aus 20 Regionen) nahmen an der Umfrage teil. Die befragten Ärzte behandeln im Schnitt 7 Skabiespatienten pro Woche, wobei eine Standardabweichung von 9 Patienten zeigt, dass hier große Unterschiede zwischen den teilnehmenden Ärzten bestehen. 


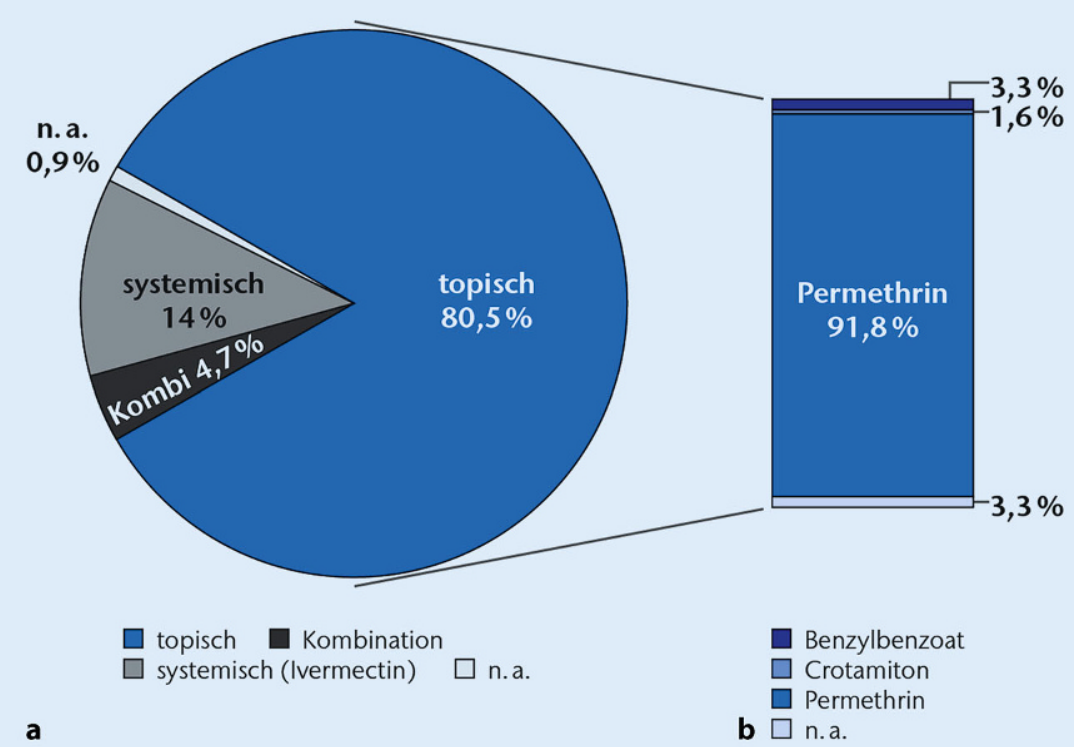

Abb. 1 A Initialtherapie der Skabies nach Applikationsart und Wirkstoff. a Gewählte Applikationsart in Prozent aller erhaltenen Antworten. b Eingesetzte topische Wirkstoffe in Prozent aller erhaltenen Antworten. n. a. nicht angegeben

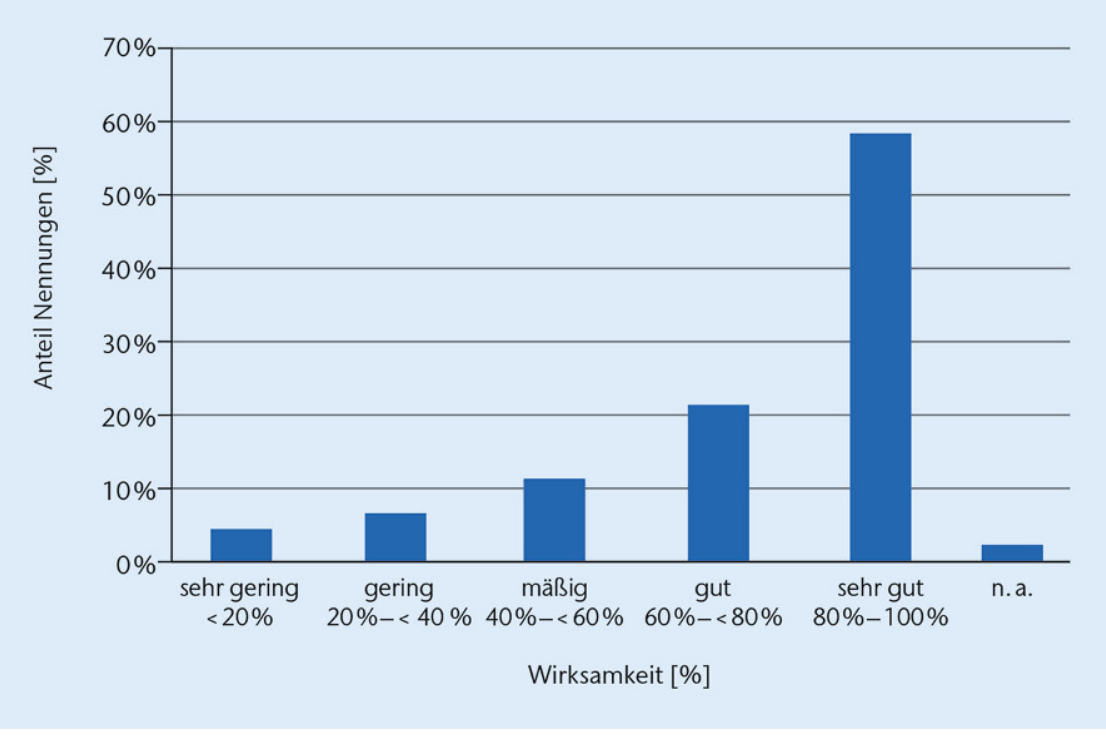

Abb. 2 \ Wahrgenommene Wirksamkeit von Permethrin. Angaben in Prozent aller erhaltenen Antworten. Die Bewertungsstufen (sehr gut, gut, mäßig, gering, sehr gering) entsprechen jeweils einem $20 \%$-Intervall. n. a. nicht angegeben

\section{Diagnostik}

Die überwiegende Zahl der Teilnehmer $(83,5 \%)$ vertraut bei der Diagnostik auf Anamnese und Beurteilung des klinischen Bildes ohne (45\%) oder mit (43,5\%) Einsatz eines Dermatoskops. Nur 11,5\% der Befragten gaben an, die Diagnose mikroskopisch zu sichern.

\section{Medikation und Wirksamkeit}

Von den Teilnehmern der Umfrage gaben $80,5 \%$ an, eine Skabies initial topisch zu behandeln. Davon verwenden 91,8\% den Wirkstoff Permethrin, 3,3\% Benzylbenzoat und 1,6\% Crotamiton. Lediglich $14 \%$ der Befragten vertrauen stattdessen auf eine systemische Initialtherapie mit Ivermectin, und $4,7 \%$ behandeln direkt mit einer Kombination aus einem topischen Wirkstoff und systemischem Ivermectin (• Abb. 1).

Die Mehrheit der teilnehmenden Ärzte $(57,2 \%)$ gibt die Wirksamkeit der topischen Permethrin-Behandlung als ,sehr gut" (80-100\%) an. Ein geringerer Teil der Befragten (19,3\%) berichtet hingegen von einer Wirksamkeit in (teilweise deutlich) weniger als $60 \%$ der Fälle und beschreibt die Erfolgsrate der Skabiestherapie mit Permethrin als „mäßig“ $(10,7 \%)$, "gering“ $(5,9 \%)$ oder „sehr gering“ (2,7\%) (• Abb. 2).

Im Mittel wird die Wirksamkeit der Permethrin-Therapie mit $73 \%$ angegeben und damit definitionsgemäßals ,gut“ bewertet.

\section{Regionale Auswertung}

Um mögliche regionale Unterschiede bei der wahrgenommenen Wirksamkeit der Therapie mit Permethrin zu identifizieren, wurden die Angaben der Teilnehmer mit dem jeweiligen Praxis- oder Klinikstandort korreliert und nach PLZ-Regionen ausgewertet. Dabei zeigte sich insgesamt ein heterogenes Bild. Während 65,3\% der Regionen im Mittel über eine "gute“ bis „sehr gute“ Wirksamkeit berichten, wurde die Wirksamkeit der Permethrin-Therapie in einigen Regionen deutlich schlechter bewertet. In 12,6\% der PLZ-Regionen wurde die Wirksamkeit als „mäßig“, in einer Region sogar als "gering“ bewertet. In keiner Region wurde eine „sehr geringe“ Wirksamkeit angegeben (• Abb. 3).

Aus 20 der bundesweit 95 PLZ-Regionen wurden keine Rückmeldungen erhalten.

In Regionen, in denen die Wirksamkeit der Permethrin-Therapie als mäßig, gering oder sehr gering $(<60 \%)$ eingeschätzt wird, zeigt sich interessanterweise eine größere intraregionale Heterogenität der Wirksamkeitseinschätzungen zwischen den einzelnen Umfrageteilnehmern als in Regionen, die die Wirksamkeit der Permethrin-Therapie als gut oder sehr gut angeben ( $\geq 60 \%)$. Im Mittel betrugen die Standardabweichungen der berichteten Wirksamkeiten der Behandlung mit Permethrin in Regionen mit einer eingeschätzten Wirksamkeit <60\% 
Hautarzt 2020 - 71:374-379 https://doi.org/10.1007/s00105-020-04561-y

(c) Der/die Autor(en) 2020

\section{B. Hackenberg · O. N. Horváth $\cdot$ M. Petachti $\cdot$ R. Schult $\cdot$ N. Yenigün $\cdot$ P. Bannenberg}

\section{Skabiestherapie in Deutschland. Ergebnisse einer bundesweiten Umfrage mit besonderem Fokus auf die Wirksamkeit der Erstlinientherapie mit Permethrin}

\section{Zusammenfassung}

Hintergrund. Therapie der ersten Wahl gemäß

Leitlinie ist bei gewöhnlicher Skabies in

Deutschland die topische Einmalbehandlung mit Permethrin $5 \%$ Creme. In den letzten Jahren gab es im Zuge zunehmender Skabiesinzidenz vermehrt Meldungen über ein Nachlassen der Wirksamkeit der Standardtherapie.

Ziel. Um ein besseres Verständnis von der Wirksamkeit der Skabiestherapie unter Praxisbedingungen zu entwickeln, wurde eine Umfrage mit besonderem Fokus auf die Erstlinientherapie mit Permethrin in dermatologischen Kliniken, Ambulanzen und Hautarztpraxen durchgeführt.

Methoden. In der postalischen Umfrage unter allen in Deutschland dermatologisch tätigen Ärzten wurden die bevorzugten Diagnoseverfahren, die jeweilige Standardinitialbehandlung, die wahrgenommene Wirksamkeit der Permethrin-Therapie, die Häufigkeit und die Gründe für ein mögliches Therapieversagen unter Permethrin und das therapeutische Vorgehen bei Versagen der Initialtherapie erfragt.

Ergebnisse. Die Wirksamkeit von Permethrin wurde von 187 Ärzten im Mittel mit 73\% bewertet. Rund $74 \%$ behandeln weiterhin initial topisch mit $5 \%$ iger Permethrin-Creme. Die am häufigsten genannten Gründe für ein Therapieversagen waren Anwendungsfehler, mangelnde Compliance und unzureichende Hygienemaßnahmen. Daneben wurde der Verdacht auf nachlassende Wirksamkeit,
Resistenzentwicklung und Re-Infestationen geäußert. Bei Unwirksamkeit der Initialtherapie entscheiden sich die meisten Ärzte für orales Ivermectin, eine Wiederholung der Permethrin-Therapie oder für eine Kombination aus beidem.

Fazit. Obwohl lokal begrenzt über ein Nachlassen der Wirksamkeit von Permethrin bei Skabies berichtet wird, deuten die Ergebnisse dieser Umfrage weiterhin auf eine gute Wirksamkeit der Erstlinientherapie mit Permethrin 5\% Creme in Deutschland hin.

Schlüsselwörter

Resistenz · Therapieversagen · Epidemiologie . Antiscabiosa · Postscabiöses Ekzem

\section{Scabies therapy in Germany. Results of a nationwide survey with a special focus on the efficacy of first- line therapy with permethrin}

\section{Abstract}

Background. According to the current scabies guideline, topical treatment with permethrin $5 \%$ cream is the first-line therapy of common scabies in Germany. However, in the course of growing incidence of scabies in recent years there have been increasing numbers of reports of reduced efficacy in standard therapy.

Objective. To establish a better understanding for the efficacy of scabies therapy under practice conditions, a survey focusing on the first-line therapy with permethrin was conducted in dermatological hospitals, outpatient clinics and dermatology practices. Methods. The postal survey addressed all dermatologically active physicians in
Germany and queried diagnostic methods, the individual initial treatment, the estimated efficacy of permethrin therapy, the frequency and reasons for therapy failures under permethrin and the therapeutic approach in case of failure of the initial therapy.

Results. The efficacy of permethrin was rated with $73 \%$ by 187 physicians. About $74 \%$ reported to treat initially with $5 \%$ permethrin cream. The most common reasons identified for treatment failure were application errors, lack of compliance and inadequate hygiene measures. Suspected diminished efficacy or development of resistance against permethrin and re-infestations were also mentioned. In the case of ineffectiveness of initial therapy, most clinicians opt for oral ivermectin, a repetition of permethrin therapy or a combination of both.

Conclusion. Although there are localized reports of decreased effectiveness of permethrin therapy, results of this survey point towards a persisting high efficacy of first-line standard treatment of scabies with permethrin $5 \%$ cream in Germany.

\section{Keywords}

Resistance - Therapy failure - Epidemiology Scabicides · Post-scabietic eczema
34,3 und in Regionen mit $\geq 60 \%$ iger Wirksamkeit nur 13,5.

\section{Therapieversagen}

Neben der wahrgenommenen Wirksamkeit wurden im Rahmen der Befragung auch die Häufigkeit und Gründe von Therapieversagen, obwohl nach Angaben der Patienten und Eindruck der behandelnden Ärzte die Anwendung korrekt durchgeführt wurde, erfragt. Insgesamt schät- zen $46 \%$ der teilnehmenden Ärzte den Anteil an Therapieversagern als niedrig (0-24\%) ein, während 25,1 \% der Ärzte ein Therapieversagen in weniger als der Hälfte der Fälle beobachten (25-49\%). Etwas mehr als ein Fünftel der Teilnehmer $(21,9 \%)$ sehen nach eigenen Angaben hingegen über $50 \%$ Therapieversager; $7 \%$ der Teilnehmer machten hierzu keine Angaben.

Die am häufigsten genannten Gründe für ein Therapieversagen waren mit
32,8\% Anwendungsfehler (Fehler bei der Anwendung durch den Patienten), gefolgt von mangelnder Compliance und unzureichenden Hygienemaßnahmen $(29,6 \%)$, Verdacht auf nachlassende Wirksamkeit oder Resistenzentwicklung $(18,8 \%)$ und Re-Infestationen (13,2\%). Als weitere Gründe für ein Therapieversagen nannten 5,6\% der Teilnehmer die Verwechslung eines postskabiösen Ekzems mit einer floriden Infestation oder „schwierige“ Patientenkollektive (z.B. 


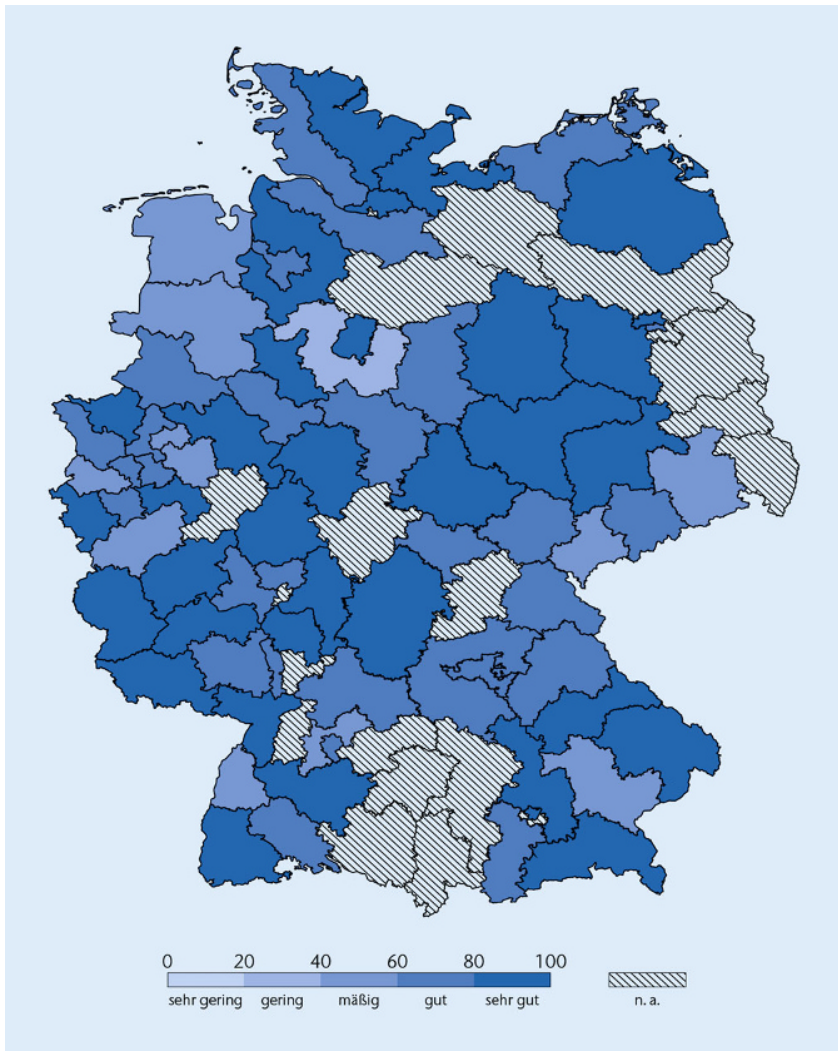

Abb. $3<$ Wahrgenommene Wirksamkeit von Permethrin zur Behandlung der Skabies in Deutschland nach Postleitzahlregionen. Dunkelblaue Regionen weisen eine hohe wahrgenommene Wirksamkeit auf, hellblaue Bereiche eine weniger hohe Wirksamkeit. Blautöne korrelieren mit den Wirksamkeitskategorien:Sehr gut $(80-100 \%)$, gut (60-80\%), mäßig (40-60\%), gering (20-40\%), sehr gering (0-20\%). Aus schraffierten Regionen liegen keine Daten vor. $n$. $a$. nicht angegeben

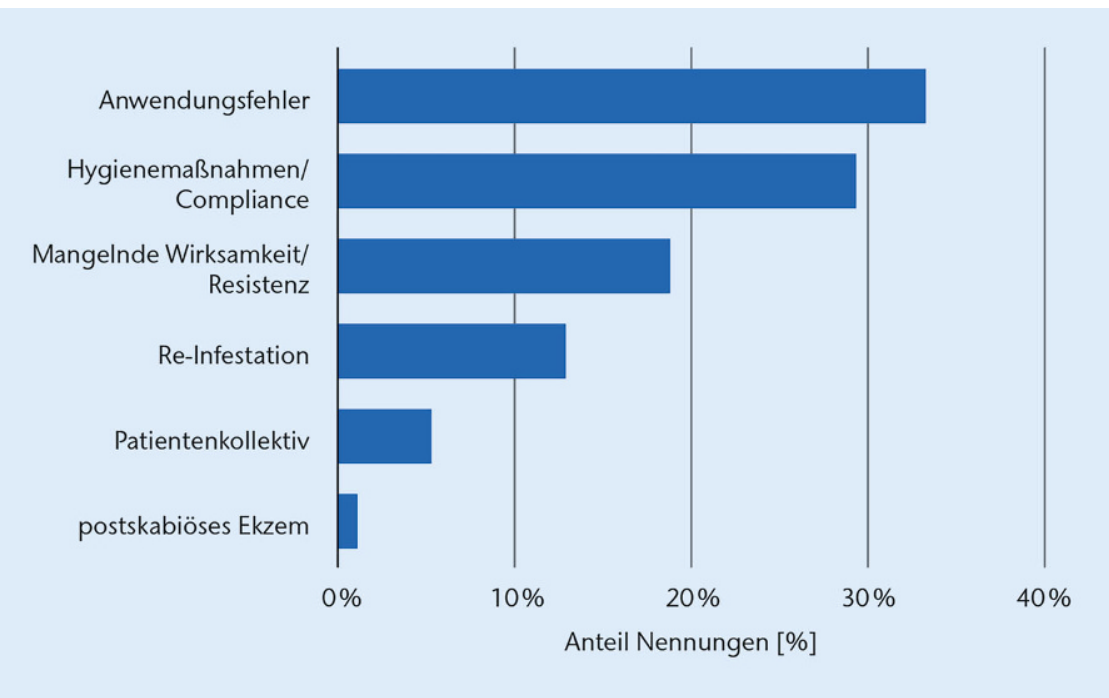

Abb. 4 \ Vermutete Gründe für ein Versagen der Skabiestherapie mit Permethrin. Angaben in Prozent aller erhaltenen Antworten

schwere Erscheinungsformen der Erkrankung, Kommunikationshürden bei der Patientenaufklärung oder auch stark übergewichtige Patienten) (• Abb.4).

\section{Maßnahmen bei Therapieversagen}

Als am häufigsten getroffene Maßnahme bei ausbleibendem Therapieerfolg mit mit anderen Wirkstoffen wie Benzylbenzoat $(7,6 \%)$ oder Crotamiton $(4,7 \%)$, ggf. in Kombination mit einer oralen Ivermectin-Therapie (jeweils 3,6 und 1,5\%), wurde dagegen nur selten angeführt (• Abb. 5).

Bei der Frage nach dem geeigneten Zeitpunkt für eine Wiederholungsbehandlung mit Permethrin bei Versagen der Initialtherapie wird von $75,9 \%$ der Teilnehmer ein Abstand von 8 bis $\geq 14$ Tagen angegeben. Nur wenige Teilnehmer der Umfrage wiederholen die Permethrin-Therapie bereits innerhalb der ersten $7(18,2 \%)$ bzw. innerhalb der ersten 5 Tage $(2,1 \%)$ nach Initialtherapie.

\section{Diskussion}

In den letzten Jahren sind Meldungen über eine nachlassende Wirksamkeit der Skabiestherapie mit Permethrin im ärztlichen Alltag häufiger geworden. Jedoch behandeln $74 \%$ der Umfrageteilnehmer initial weiterhin leitlinienkonform und erfolgreich mit Permethrin 5\% Creme. Die dabei in der Praxis wahrgenommene Wirksamkeit von $73 \%$ widerspricht nur auf den ersten Blick den Ergebnissen früherer Untersuchungen. So wurde im Rahmen der pivotalen multizentrischen Studie über eine klinische Heilungsrate von $95 \%$ bei Anwendung von Permethrin $5 \%$ Creme berichtet, während ein aktueller Cochrane-Review eine mittlere Wirksamkeit von $93 \%$ (nach 1 bis 3 Behandlungen) beschreibt $[6,7]$. Im Gegensatz hierzu beziehen sich die aktuellen Umfrageergebnisse zur Wirksamkeit auf die Permethrin-Therapie in der Praxis, die im Vergleich zur Therapie unter optimierten Bedingungen einer kontrollierten klinischen Studie einer Vielzahl von Limitationen unterworfen ist. So können beispielsweise die von den Teilnehmern der Umfrage am häufigsten genannten Gründe für ein Versagen der Permethrin-Initialtherapie (Anwendungsfehler, mangelnde Hygienemaßnahmen/Compliance) in einer klinischen Studie weitestgehend ausgeschlossen werden.

Entsprechend sehen $47,1 \%$ der befragten Ärzte trotz einer insgesamt als "gut" (73\%) empfundenen Wirksamkeit der Permethrin-Behandlung mindes- 


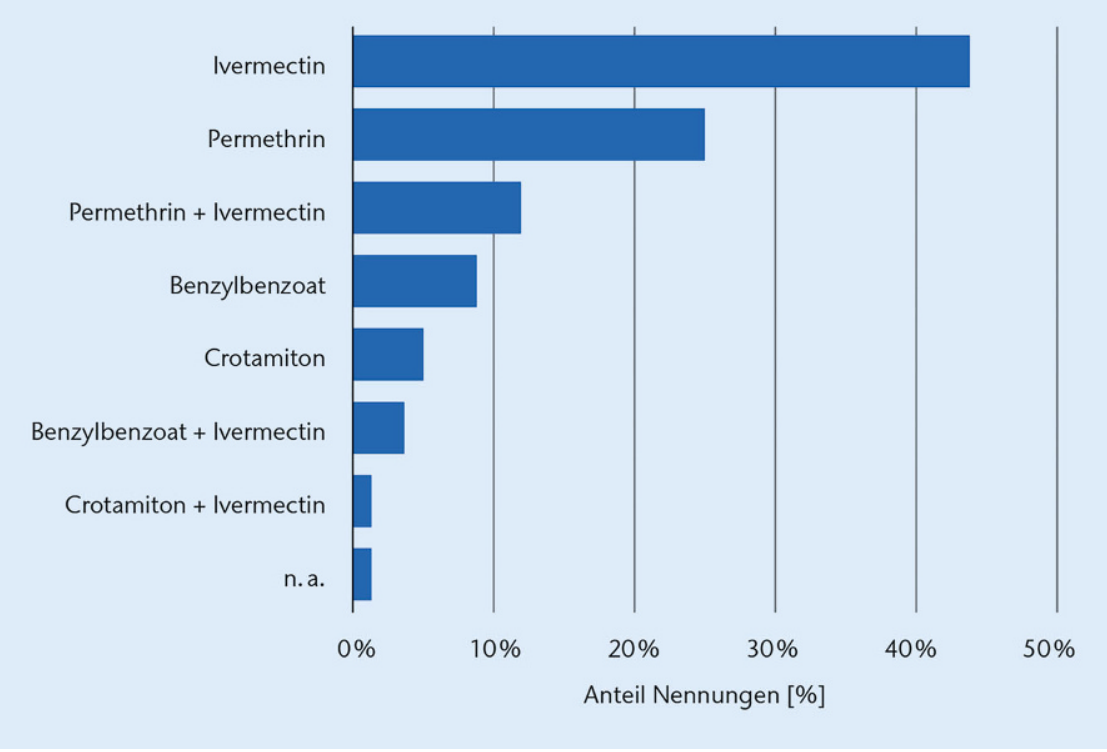

Abb. 5 - Maßnahmen bei Versagen der Skabiestherapie mit Permethrin. Angaben in Prozent aller erhaltenen Antworten. n. a. nicht angegeben

tens $25 \%$ und teilweise sogar über $50 \%$ Therapieversager. Die von $75,6 \%$ der befragten Dermatologen vermuteten Gründe für Therapieversagen umfassen neben den bereits erwähnten Anwendungsfehlern durch den Patienten und einer nicht sachgerechten Durchführung von Hygiene- und Umgebungsmaßnahmen auch Fehler bei der Mitbehandlung von Kontaktpersonen sowie Re-Infestationen. Diese Einschätzung deckt sich mit den Ergebnissen eines aktuellen Reviews zu den Hintergründen und Konsequenzen der aktuellen Zunahme von Skabiesfällen in Deutschland, wonach v. a. Anwendungsfehler und ReInfestationen zu Therapieversagen in der Praxis führen [8]. Die Autoren betonen die Notwendigkeit einer umfassenden Patientenaufklärung und raten dazu, Informationsmaterial in verschiedenen Fremdsprachen bereitzuhalten, um die sachgerechte Anwendung z. B. von Permethrin-Creme sowie eine ausreichende Umgebungsbehandlung (Hygiene, Kontaktpersonen) sicherzustellen.

Neben den genannten Gründen wurden von einigen Teilnehmern $(18,8 \%)$ auch Milben-intrinsische Faktoren (zunehmende Unempfindlichkeit gegenüber Permethrin bzw. Resistenz) als Gründe für ein Therapieversagen vermutet. Denkbare Resistenzmechanismen der Krätzemilbe gegenüber Permethrin aus In-vitro-Versuchen beziehen sich auf eine „Knockdown“-Resistenz (kdrMutation) in der Zielstruktur des Wirkstoffes, d.h. im Natrium-Kanal-Gen von Skabiesmilben [9], während andere Mutationen auch eine beschleunigte Elimination von Permethrin durch Effluxpumpen oder einen gesteigerten Abbau durch Esterasen bzw. andere Entgiftungsenzyme bewirken könnten [10, 11]. Zwar konnte eine erhöhte Toleranz der humanen Krätzemilben gegenüber Permethrin in einer prospektiven Studie in vitro nachgewiesen werden [12], bislang gibt es jedoch keine Berichte, die eine In-vivo-Resistenz der Spezies gegenüber Permethrin bestätigen. Interessanterweise konnte eine molekulare Resistenzuntersuchung aus Frankreich an isolierten Milben von 40 Skabiespatienten bei keinem der untersuchten Erreger eine kdr-Mutation nachweisen [13].

Auch für Deutschland gibt es aktuell keine eindeutigen Hinweise auf eine bestehende Resistenzsituation der Krätzemilbe Sarcoptes scabiei var. hominis gegenüber Permethrin [9]. Im Rahmen der vorliegenden Umfrage wurde von der Mehrzahl der PLZ-Regionen weiterhin über eine gute bis sehr gute wahrgenommene Wirksamkeit $(\geq 60 \%)$ von Permethrin berichtet. Unabhängig von den möglichen Gründen lässt die re- gionale Auswertung der Angaben darauf schließen, dass das von einigen Teilnehmern geschilderte Therapieversagen eher ein räumlich begrenztes, lokales Phänomen als ein überregionales Problem darstellt. Diese Vermutung wird durch die Beobachtung gestützt, dass innerhalb von PLZ-Regionen, in denen im Durchschnitt eine geringe Permethrin-Wirksamkeit angegeben wurde, oftmals sehr unterschiedliche Wirksamkeitsraten berichtet wurden. Exemplarisch können an dieser Stelle die PLZ-Region 26 (Oldenburg, Wilhelmshaven, Emden, Aurich) und die PLZ-Region 49 (Osnabrück, Melle, Ibbenbüren, Lingen) angeführt werden. Aus der PLZ-Region 26 wurden 2 ausgefüllte Fragebögen mit 2\% bzw. $92,5 \%$ berichteter Wirksamkeit eingesandt, während aus der PLZ-Region 49 Wirksamkeiten von 0,65 und $75 \%$ berichtet wurden. Insbesondere in PLZ-Regionen mit einer geringen berichteten Permethrin-Wirksamkeit zeigte sich ein sehr heterogenes Bild der Wirksamkeitseinschätzungen selbst von Praxen und Kliniken in unmittelbarer räumlicher Nähe. Die Gründe hierfür lassen sich anhand der Ergebnisse dieser Umfrage nicht abschließend klären.

Bei Therapieversagen der (ggf. wiederholten) Permethrin-Anwendung wechselt der größte Teil der Ärzte auf eine orale Therapie mit Ivermectin, was im Wesentlichen den Empfehlungen der deutschen Leitlinie entspricht, oder kombiniert die topische Therapie mit oralem Ivermectin. Der ermittelte Anteil an Kombinationsbehandlungen von 12,7\% könnte in der Praxis höher ausfallen, da im Rahmen der Umfrage eine Kombinationstherapie nur dann angenommen wurde, wenn diese auch explizit als solche gekennzeichnet war (z. B. „Kombinationstherapie mit Permethrin und Ivermectin“). Eine reine Auflistung der Wirkstoffe (z. B. ,Therapie mit Permethrin, Ivermectin") wurde als 2 Monotherapieoptionen gewertet. Kombinationstherapien sind in der deutschen Leitlinie nicht beschrieben (Ausnahme: Scabies crustosa), werden jedoch z.B. in Österreich von der Österreichischen Gesellschaft für Dermatologie und Venerologie (ÖGDV) sowie der Österreichischen Gesellschaft für STD und dermatologische Mikrobiolo- 
gie (ÖGSTD) empfohlen [14]. Weiterhin behandeln die Teilnehmer der Umfrage zum Teil trotz initial erfolgloser Permethrin-Therapie nochmals topisch mit Permethrin. Eine Wiederholungsbehandlung wird von $75,9 \%$ der Umfrageteilnehmer im Abstand von 8 bis $\geq 14$ Tagen zur Initialtherapie durchgeführt, was im Wesentlichen den Empfehlungen der Fachinformation des zugelassenen Fertigarzneimittels (InfectoScab ${ }^{\circledR} 5 \%$ Creme [InfectoPharm Arzneimittel und Consilium $\mathrm{GmbH}$, Heppenheim, Deutschland] Wiederholungstherapie nach 14 Tagen möglich) bzw. der deutschen Leitlinie (Wiederholungstherapie nach $7 / \geq 14$ Tagen) entspricht [1].

Limitiert wird die Aussagekraft der vorliegenden Daten durch die Methodik der Datenerhebung (ungestützte, retrospektive Umfrage innerhalb eines zufälligen, ggf. trotz der großen Zahl und guten bundesweiten Verteilung der Teilnehmer nicht hinreichend repräsentativen Kollektivs) sowie die fallunabhängigen und subjektiven Angaben der teilnehmenden Ärzte. Interessanterweise lassen sich die Ergebnisse zur Wirksamkeit der Permethrin-Erstlinientherapie (Mittelwert: 73\%) dennoch mit den Ergebnissen klinischer Studien der vergangenen 25 Jahre in Einklang bringen. Hier wurde im Mittel über eine Eradikationsrate von $74 \% 2$ Wochen nach Initialtherapie berichtet, während die Wirksamkeit gemessen an der Heilungsrate 4 Wochen nach 1-bis 3-maliger Permethrin-Therapie bei $93 \%$ lag [7]. Für eine belastbarere Aussage über die Wirksamkeit der Erstlinientherapie mit Permethrin und die Gründe für eine lokal beobachtete verminderte Wirksamkeit sind weitere Untersuchungen notwendig.

\section{Fazit für die Praxis}

- Von den Umfrageteilnehmern behandeln $74 \%$ eine Skabies initial leitlinienkonform mit Permethrin 5\% Creme.

- Die Ärzte geben die Wirksamkeit dieser Therapie im Mittel mit 73\% und damit definitionsgemäß mit "gut" an.
- Auffallend sind die großen inter- und sogar intraregionalen Unterschiede in der Bewertung der Wirksamkeit von Permethrin, die aktuell keine Hinweise auf ein flächendeckendes Nachlassen der Wirksamkeit liefern, jedoch auf lokal begrenzte Probleme bei der Permethrin-Therapie hinweisen.

- Bei Therapieversagen wechseln die befragten Ärzte am häufigsten $(43,1 \%)$ zu oralem Ivermectin, oder sie wiederholen die leitlinienkonforme Standardtherapie mit Permethrin $5 \%$ Creme $(25,4 \%)$.

\section{Korrespondenzadresse}

\section{P. Bannenberg}

InfectoPharm Arzneimittel und Consilium $\mathrm{GmbH}$

Von-Humboldt-Str. 1, 64646 Heppenheim, Deutschland

paul.bannenberg@infectopharm.com

\section{Einhaltung ethischer Richtlinien}

Interessenkonflikt. P. Bannenberg ist Mitarbeiter der Firma InfectoPharm Arzneimittel und Consilium GmbH, Heppenheim. B. Hackenberg, O.N. Horváth, M. Petachti, R. Schult und N. Yenigün geben an, dass kein Interessenkonflikt besteht.

Für diesen Beitrag wurden von den Autoren keine Studien an Menschen oder Tieren durchgeführt. Für die aufgeführten Studien gelten die jeweils dort angegebenen ethischen Richtlinien.

Open Access. Dieser Artikel wird unter der Creative Commons Namensnennung 4.0 International Lizenz veröffentlicht, welche die Nutzung, Vervielfältigung, Bearbeitung, Verbreitung und Wiedergabe in jeglichem Medium und Format erlaubt, sofern Sie den/die ursprünglichen Autor(en) und die Quelle ordnungsgemäßnennen, einen Link zur Creative Commons Lizenz beifügen und angeben, ob Änderungen vorgenommen wurden.

Die in diesem Artikel enthaltenen Bilder und sonstiges Drittmaterial unterliegen ebenfalls der genannten Creative Commons Lizenz, sofern sich aus der Abbildungslegende nichts anderes ergibt. Sofern das betreffende Material nicht unter der genannten Creative Commons Lizenz steht und die betreffende Handlung nicht nach gesetzlichen Vorschriften erlaubt ist, ist für die oben aufgeführten Weiterverwendungen des Materials die Einwilligung des jeweiligen Rechteinhabers einzuholen.

Weitere Details zur Lizenz entnehmen Sie bitte der Lizenzinformation auf http://creativecommons.org/ licenses/by/4.0/deed.de.

\section{Literatur}

1. Sunderkötter $C$, Feldmeier $H$, Fölster-Holst R et al (2016) S1-Leitlinie zur Diagnostik und Therapie der Skabies. AWMF online 2016, Reg.-Nr. 013-052. JDtsch Dermatol Ges 14(11):1160-1171

2. Insight Health Galaxy, NPI-Daten - Verkaufte Antiscabiosa in Deutschland 2012-2018, Abfrage 07/2019

3. Kämmerer E (2018) Skabies: Erfahrungen aus der Praxis. Dtsch Arztebl 115(15):A-700/B-604/C-605

4. Dressler $C$, Rosumeck $S$, Sunderkötter $C$ et al (2016) The treatment of scabies. Dtsch Arztebl Int 113(45):757-762

5. IQVIA OneKey Health Care Professionals Datenbank, Fachgruppe Dermatologie, Stand 09/2019: 7.651 dermatologisch tätige Ärzte.

6. Hamm H, Beiteke U, Höger PH et al (2006) Treatment of scabies with $5 \%$ permethrin cream: results of a German multicenter study. J Dtsch Dermatol Ges 4(5):407-413

7. Rosumeck S, Nast A, Dressler C (2018) Ivermectin and permethrin for treating scabies. Cochrane Database Syst Rev. https://doi.org/10.1002/ 14651858.CD012994

8. Sunderkötter C, Aebischer A, Neufeld Met al (2019) Zunahme von Skabies in Deutschland und Entwicklung resistenter Krätzemilben? Evidenz und Konsequenz. JDtsch Dermatol Ges 17(1):15-24

9. Pasay C, Walton S, Fischer K et al (2008) High resolution melt analysis for the detection of a mutation associated with permethrin resistance in a population of scabies mites. Med Vet Entomol 22(1):82-88

10. Mounsey KE, Pasay CJ, Arlian LG et al (2010) Increased transciption of glutathione $S$-transferases in acaricide exposed scabies mites. Parasit Vectors 3:43

11. Pasay C, Arlian L, Morgan M et al (2009) The effect of insecticide synergists on the response of scabies mites to pyrethroid acaricides. PLoS Negl Trop Dis 3(1):e354

12. Walton SF, Myerscough MR, Currie BJ (2000) Studies in vitro on the relative efficacy of current acaricides for Sarcoptes scabiei var hominis. Trans RSoc Trop Med Hyg 94(1):92-96

13. Andriantsoanirina V, Izri A, Botterel F et al (2014) Molecular survey of knockdown resistance to pyrethroids in human scabies mites. Clin Microbio Infect 20(2):0139-0141

14. Österreichische Gesellschaft für Dermatologie und Venerologie (ÖGDV), Österreichische Gesellschaft für STD und dermatologische Mikrobiologie (ÖGSTD) (2019) Skabies Therapiemanagement für Allgemeinmediziner und Fachärzte. http:// oegstd.at/uploads/Skabies_therapie_11-2019. pdf.Zugegriffen: 26.02.2020 